\title{
Can Left-Handedness be Switched? Insights from an Early Switch of Handwriting
}

\author{
Stefan Klöppel, ${ }^{1,2,4}$ Anna Vongerichten, ${ }^{1}$ Thilo van Eimeren, ${ }^{1,3}$ Richard S. J. Frackowiak, ${ }^{2,5,6}$ and Hartwig R. Siebner ${ }^{1,7}$ \\ ${ }^{1}$ NeuroImage-Nord, Hamburg-Lübeck-Kiel, Germany, ${ }^{2}$ Wellcome Trust Centre for Neuroimaging, University College London, London WC1N 3BG, United \\ Kingdom, ${ }^{3}$ Department of Neurology, University Medical Center Hamburg-Eppendorf, 20246 Hamburg, Germany, ${ }^{4}$ Department of Neurology, \\ Neurozentrum, University Clinic Freiburg, 79106 Freiburg, Germany, ${ }^{5}$ Laboratory of Neuroimaging, IRCCS Santa Lucia, 00179 Rome, Italy, ${ }^{6}$ Departement \\ d'Etudes Cognitives, Ecole Normale Superieure, 75005 Paris, France, and 7Department of Neurology, Christian-Albrechts-University, 24105 Kiel, Germany
}

\begin{abstract}
"Converted" left-handers are innately left-handed individuals forced as children to write with the right nondominant hand. We asked how a left-to-right handwriting switch shapes cortical sensorimotor representations of finger movements. In 16 adult converted lefthanders and age-matched groups of 16 consistent right-handers and 16 left-handers, we studied movement-related neuronal activity with functional magnetic resonance imaging while participants performed simple unimanual and bimanual movements with right and left index fingers. In converted left-handers, movement-related activity in the primary sensorimotor hand area (SM1) and caudal dorsal premotor cortex (PMd) of the nondominant left hemisphere correlated with the left-to-right shift in handedness. The more right-handed converted left-handers had become, the greater the sensorimotor activation in these areas. Between-group comparisons showed that the switch from left to right hand also reinforced movement representations in the dominant right hemisphere. In converted left-handers, the right inferior parietal cortex and lateral PMd were more active relative to consistent right or left-handers in all motor tasks. These results suggest two distinct neuronal correlates of handedness in human sensorimotor cortex. Although those in executive sensorimotor cortex (i.e., SM1 and adjacent PMd) depend on the hand used throughout life, those in higher-order sensorimotor areas (i.e., inferior parietal cortex and rostrolateral PMd) are invariant and thus cannot be switched to the nondominant hemisphere by educational training.
\end{abstract}

Key words: handedness; converted left-handers; fMRI; motor control; parietal; plasticity

\section{Introduction}

Most humans exhibit some degree of handedness. In contrast to other primates, the majority of humans prefer the right hand for skilled manipulation. Independent of cultural background, $\sim 90 \%$ of humans are right handed (Corballis, 2003). Although handedness has been attributed to genetic factors (Sun and Walsh, 2006), the exact basis and neuronal correlates remain to be determined. Several postmortem (Witelson and Kigar, 1992; White et al., 1994; Amunts et al., 1996), structural (Amunts et al., 2000; Good et al., 2001; Büchel et al., 2004; Herve et al., 2005, 2006), and functional magnetic resonance imaging (fMRI) studies (Kim et al., 1993; Dassonville et al., 1997; Singh et al., 1998; Solodkin et al., 2001; Klöppel et al., 2007) have shown structural and functional hemispheric asymmetries between adult rightand left-handers (for review, see Serrien et al., 2006; Sun and Walsh, 2006). However, on the basis of these studies, it is impossible to infer whether handedness-related differences are deter-

Received Nov. 24, 2006; revised June 9, 2007; accepted June 11, 2007.

This work was supported by grants from the BMBF (Ministry for Education and Science; (01 G0 0510, 01 G0 0511 , and $01 \mathrm{G0} 0513$ to H.R.S.), the Volkswagenstiftung (I/79-932 and I/78-553 to H.R.S.), and the Wellcome Trust (075696 2/04/2 to R.S.J.F.). We thank Dr. Barbara Sattler ("Consulting agency for left-handers," Munich, Germany) for her support to recruit "converted" left-handers.

Correspondence should be addressed to Dr. Stefan Klöppel, Wellcome Trust Centre for Neuroimaging, 12 Queen Square, London WC1N 3BG, UK. E-mail: s.kloppel@fil.ion.ucl.ac.uk.

D0I:10.1523/JNEUROSCI.1299-07.2007

Copyright $\odot 2007$ Society for Neuroscience $\quad$ 0270-6474/07/277847-07\$15.00/0 minant or reflect a long-term consequence of actual hand use. To resolve this ambiguity, we studied primarily left-handed individuals forced as children to write with their right hand. "Converted" left-handers switched to right-handedness for writing usually continue to preferentially use the left hand for many other skilled manual activities.

Using positron emission tomography (PET) and normalized regional cerebral blood flow, we previously compared neuronal activity during right-handed writing in converted left-handers and natural right-handers (Siebner et al., 2002). Converted lefthanders showed a relative increase in writing-related activity in the dominant right lateral premotor, inferior parietal, and temporal cortices. Writing-related neuronal activity in right rostral supplementary motor area and inferior parietal lobule correlated with the degree of left-handedness. Although this study demonstrated persistent differences in the functional neuroanatomy of handwriting between adult right-handers and converted lefthanders, it provided no information about how switching handedness for writing shapes the functional neuroanatomy of other manual actions. We therefore examined whether an early switch of handwriting triggers a more generalized motor reorganization. This should be manifest during motor tasks not switched by educational training. Using fMRI, we investigated how a left-toright switch in handwriting during childhood impacts on effector-independent neuronal representations of simple button presses with the right, left, or bilateral index finger. 


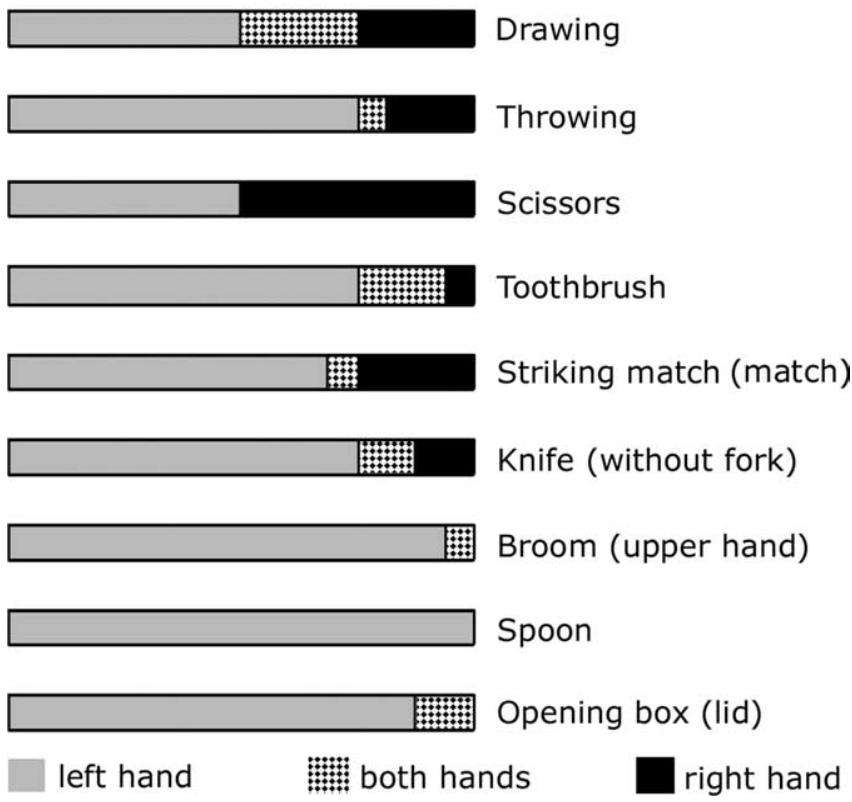

Figure 1. Distribution of right- and left-hand preference for each item of the Edinburgh handedness score in the group of converted left-handers.

Our primary hypothesis was that a switch of handwriting results in lateralized reorganization of other cortical motor representations that subserve skilled as well as simple hand movements. If so, the degree of conversion across individuals should correlate with the amount of neuronal activation engaged by other simple hand movements. We tested three alternative hypotheses. (1) In case of a successful switch, there will be no significant difference in movement representations between righthanders and converted left-handers. (2) If the conversion is only partially successful, there will be a shift in motor representations from left-handed patterns to the pattern found in right-handed subjects (i.e., shifted to the left hemisphere). (3) If hand preference is firmly imprinted in cortical motor areas of the right hemisphere in left-handers, a handedness switch will reinforce motor representations in the dominant right hemisphere.

\section{Materials and Methods}

Participants. Sixteen converted left-handers (mean age, 36.4 years; range, 24-47 years; 9 males) were compared with 16 consistent age- and sexmatched right-handed subjects (mean age, 33.3 years; range, 24-57 years; 10 males) and 16 left-handed subjects (mean age, 35 years; range, 25-56 years; 10 males). Converted left-handers were recruited via advertisement in which we asked for volunteers who showed an early and consistent preference for left-handed movements at preschool age (4-6 years of age) but were forced to learn handwriting with their right nondominant hand. Accordingly, all converted left-handers included in the study reported a clear preference for the left hand on commencing school. They or their parents clearly recalled them starting writing with the left hand at school with a subsequent switch to the right. None of the converted left-handers were members of the cohort we examined in our previous PET study (Siebner et al., 2002). Data from 13 consistent righthanded and 14 left-handed subjects are reported from a previous study that used the same paradigm (Klöppel et al., 2007).

Handedness was determined with the Edinburgh handedness questionnaire (Oldfield, 1971). This results in a laterality quotient in which a score of -100 reflects extreme left-handedness and +100 reflects extreme right-handedness. Scores ranged from -80 to 0 (mean laterality quotient, -43.4 ) in the converted group (Fig. 1). The two groups with consistent handedness reported scores from 60 to 100 (mean, 94.2) and from -60 to -100 (mean, -83.8 ), respectively (supplemental Fig. S1, available at www.jneurosci.org as supplemental material). In addition, handedness was assessed by asking participants to pantomime six manual tasks [writing, throwing, holding a tennis racket, striking a match (hand holding match), holding a hammer, brushing teeth] as listed in Annett's handedness questionnaire (Annett, 1976). Participants were labeled as consistent right- or left-handers when all six items were pantomimed with the same (dominant) hand. Participants gave written informed consent to the study. Experimental procedures were approved by the local Ethics Committee and performed according to the ethical standards laid down in the Declaration of Helsinki.

Handwriting task outside the MR scanner. Participants were asked to continuously write pairs of two lower case "l" letters ("ll") for a $10 \mathrm{~s}$ period (see Fig. 2, left) at normal speed. All subjects performed the task with both right and left hands. The handwriting task was used to obtain a measure of manual dexterity and to check whether right-handed handwriting was matched between converted left-handers and consistent right-handers. We chose a relatively simple writing task because it could be performed with both the preferred and nonpreferred hands.

To obtain an objective measure of handwriting, writing was continuously recorded with a digitizing graphics tablet and an inking digitizing pen (UD-1212; WACOM Europe, Neuss, Germany). The position of the pen tip was stored on a personal computer at a sample frequency of 166 $\mathrm{Hz}$. The spatial resolution was $0.05 \mathrm{~mm}$. Kinematic analysis used a personal computer-based program (CS software; MedCom, Munich, Germany). Kinematic analyses were based on writing movements along the vertical axis because this is the axis along which writing movements predominantly occur (Marquardt and Mai, 1994). The written trace was automatically segmented into consecutive up-and-down strokes. Curves of vertical position and velocity were calculated and smoothed by nonparametric regression methods. The mean frequency of consecutive upand-down strokes was calculated for handwriting with the right and left hand. This measure is a well established marker of the degree of automation of handwriting (Marquardt and Mai, 1994).

Choice reaction-time task during $f M R I$. Participants performed a visually cued choice reaction-time task with their right, left, or both index fingers in response to a symbolic cue. Each trial started with the presentation of a cue in the center of the visual field. The cue was presented for $1.0 \mathrm{~s}$, and the time between two consecutive cues was jittered between 2.0 and $2.3 \mathrm{~s}$. By choosing symmetrical stimuli, avoiding any directional information, and by presenting stimuli sequentially in the center of the screen, we tried to minimize shifts in spatial attention. Symbols were light gray in color presented on a dark gray background (see Fig. $3 A$ ). There were three symbolic cues, each instructing a different movement (pressing a button with the left, right, or both index fingers). Responses were made as quickly as possible with two buttons set beneath the right or left index fingers. For a bilateral motor response, participants pressed both buttons simultaneously. A fourth cue instructed participants to keep still (referred to as a no-go condition). The no-go condition was introduced to prevent response anticipation. The assignment of cues to tasks was counterbalanced across participants. Each of the four cues was presented 48 times per fMRI run. In addition, there were 48 null events without cue presentation. The order of cue presentation and null events was pseudorandomized. Participants had no advance information about movements to be made in successive trials. The software package PRESENTATION (Neurobehavioral Systems, Albany, NY) was used for stimulus presentation, synchronization of stimuli, image pulse acquisition, and recordings of motor responses during fMRI. In each trial, stimulus onset and reaction time were extracted from the results file. Mean reaction time was calculated for correct responses of each trial type. For trials that required bilateral responses, we used the first button press to calculate mean reaction time. To correlate performance in the handwriting task with imaging data, a quotient (mean stroke frequency right hand/mean stroke frequency left hand) was computed. Values $>1$ indicate lateralization to the right. The visuomotor reaction-time task was suitable for probing effector-independent sensorimotor representations because the task required unilateral and bilateral responses with right and left hands. A second reason for choosing a relatively simple reaction-time task was to 
avoid any differential task performance related to individual expressions of handedness (Klöppel et al., 2007). We refrained from using a more skillful manual task because differential right-left performance is a usual finding in such tasks. We were concerned that such differences would confound the interpretation of task-related group differences in neuronal activity (Stucchi et al., 1993; Lutz et al., 2005).

fMRI. MRI was performed on a 3T system (TRIO; Siemens, Erlangen, Germany). We used single-shot gradient-echo echo-planar imaging (EPI) [repetition time (TR), $2.12 \mathrm{~s}$; echo time (TE), $25 \mathrm{~ms}$; flip angle, $70^{\circ}$ ] to measure task-related blood oxygenation level-dependent (BOLD) signal changes (used as an index of regional brain activity). A single brain volume consisted of 37 transversally oriented slices (slice thickness, 3 $\mathrm{mm}$; matrix, $192 \times 192 \mathrm{~mm}$; in-plane resolution, $3 \times 3 \mathrm{~mm}$ ). The field of view covered both cerebral hemispheres. Three hundred brain volumes were acquired in a single fMRI session lasting $\sim 10 \mathrm{~min}$.

We excluded structural abnormalities in the brain with a T1-weighted FLASH three-dimensional whole-brain sequence (TR, $15 \mathrm{~ms}$; TE, $4.92 \mathrm{~ms}$; flip angle, $25^{\circ}$; 192 slices; slice thickness, $1 \mathrm{~mm}$; matrix, $256 \times 256 \mathrm{~mm}$ ).

Behavioral data analysis. Separate repeated-measures ANOVA were computed to examine the mean frequency of up-and-down strokes during handwriting and mean reaction times during the visually cued reaction-time motor task. Both measures were treated as dependent variables in a two-factorial ANOVA with a within-subject factor, "performing hand" (two levels: right and left), and a between-subjects factor, "handedness" (three levels: right-handers, left-handers, and converted left-handers). Conditional on significant $F$ test results, we performed post hoc pairwise comparisons using the Scheffe's test. In the reaction-time task, we also assessed how often participants made first button presses with left and right hands, respectively. The significance level was set at $p<0.05$. Nonparametric tests were used to compare sex, percentage of correct button presses, and percentage of button presses with each hand in the bilateral condition. Behavioral data are given as mean \pm SD for parametric tests and as median \pm range for nonparametric data.

Imaging data analysis. Preprocessing and statistical analysis of the fMRI data were performed using SPM2 software (www.fil.ion.ucl.ac.uk/ $\mathrm{spm} /$ ). The first three volumes of each session were excluded from data analysis because of magnetization effects. The remaining volumes were realigned to the first volume and spatially normalized to a standard EPI template in Montreal Neurological Institute (MNI) space. The images were then spatially smoothed with an isotropic Gaussian kernel of $8 \mathrm{~mm}$ full-width at half-maximum to allow statistical inference using the Gaussian random-field theory and temporally filtered with a high-pass filter to remove baseline drifts.

A first-level analysis based on the general linear model (Friston et al., 1995) was performed for each subject individually. Task-related changes in BOLD signal were estimated at each voxel by modeling the onsets of each trial as $\delta$ functions convolved with a hemodynamic response function (HRF). Each of the four experimental conditions (right button press, left button press, bilateral button press, and "no-go" condition) and incorrect responses were modeled as separate regressors. In addition, we included six regressors obtained at the realignment step to account for movements (translations in three planes and rotations along three axes). Null events were not modeled and provided the baseline for conditionspecific main effects. Condition-specific effects were tested using the appropriate linear contrasts of parameter estimates for the HRF regressors of all conditions. The resulting set of voxel values for each contrast constitutes a statistical parametric map.

For group inferences (second level), we performed a random-effects analysis treating subjects as a random variable. The individual parameter images corresponding to each movement condition, derived from the first-level analysis, were entered into separate between-group ANOVAs that used the condition-specific BOLD signal changes as a dependent measure. Thus, each ANOVA model contained the parameter images of only one movement condition (i.e., right, left, or bilateral button presses) and three groups (converted and consistent left-handers, consistent right-handers). Within each ANOVA model, we specified appropriately weighted linear contrasts to test for regional differences in movementrelated activation between the three groups with an $F$ test.

Our primary aim was to identify brain regions where converted left- handers show a consistent difference in task-related BOLD signal changes independent of the effector, and therefore across all movement conditions, relative to individuals with consistent right- and lefthandedness. To this end, we performed a stepwise masking approach. This procedure enabled us to use fully orthogonal contrasts to effectively reduce the search volume and thereby increase sensitivity. The statistical images containing the $F$ values (F-maps) obtained in two separate ANOVAs were combined to define a region of interest for the F-map obtained in the third ANOVA. F-maps that were used to generate the masks were thresholded at $p<0.01$ (uncorrected). For instance, the F-map showing voxels with differential activity for unilateral button presses between all three groups was used to generate an inclusive mask for the F-map showing differential activity for bilateral button presses. The order of masking did not change the significance of results. To avoid any bias toward a positive finding, we report the results for the masking order that gave the lowest $F$ score (for full details, see supplemental Table 1, available at www.jneurosci.org as supplemental material).

For peak voxels in regions, identified by $F$ statistics, which showed effector-independent, differential, movement-related BOLD signals in the three groups, we performed post hoc $t$ tests. Post hoc comparisons tested for significant differences in task-related BOLD signal changes between converted left-handers and consistent left-handers as well as converted left-handers and consistent right-handers. For completeness, we also report results of a comparison between the two groups with consistent handedness (supplemental Table S2, available at www.jneurosci.org as supplemental material).

In converted left-handers, we performed an additional within-group analysis to identify areas in which task-related neuronal activity correlated positively and linearly with degree of rightward shift in hand preference. This analysis was performed in the converted group only because the aim was to demonstrate a relationship between neuronal activation evoked by simple button presses and degree of switching. We performed two separate regressions. Individual laterality quotients derived from Edinburgh handedness scores and handwriting stroke frequencies were used as explanatory variables with the task-related change in BOLD signal as the dependent variable. We were interested to identify brain voxels that showed an effector-independent relationship between the degree of conversion and task-related activity across all three types of response. Therefore, we used a similar stepwise masking approach as for the categorical comparison described above. A separate simple regression model with a behavioral measure as explanatory variable (writing quotient or Edinburgh score) was tested separately for each movement type. Voxels correlating significantly in any two conditions (threshold: $p<0.01$, uncorrected) generated a mask to constrain the search volume for a correlation with the third conditions. We report results with the masking order that gave the lowest $T$ scores (for full details, see supplemental Table 3, available at www.jneurosci.org as supplemental material).

Results were corrected for multiple nonindependent comparisons within a volume of interest or for the entire brain if no mask was applied. We used the false discovery rate correction as implemented in SPM2 at $p<0.05$. All imaging results are reported with corresponding significance levels at peak coordinates in MNI space.

\section{Results}

\section{Behavioral results}

Using stroke frequency as a dependent variable, repeatedmeasures ANOVA revealed a main effect of hand $\left(F_{(1,45)}=6.20\right.$; $p=0.017)$ and an interaction between hand and handedness $\left(F_{(2,45)}=175.42 ; p<0.001\right)$. The interaction indicates a difference in mean stroke frequency between hands that depends on handedness. The handwriting task showed major asymmetries in writing speed between dominant and nondominant hands in consistent right- and left-handers (Fig. 2). Post hoc tests showed differences between consistent left-handers and both other groups with either hand. Consistent left-handers performed faster when they used their left dominant hand, whereas consistent right-handers and converted left-handers wrote faster with their right hand relative to the left hand. No differences in mean 


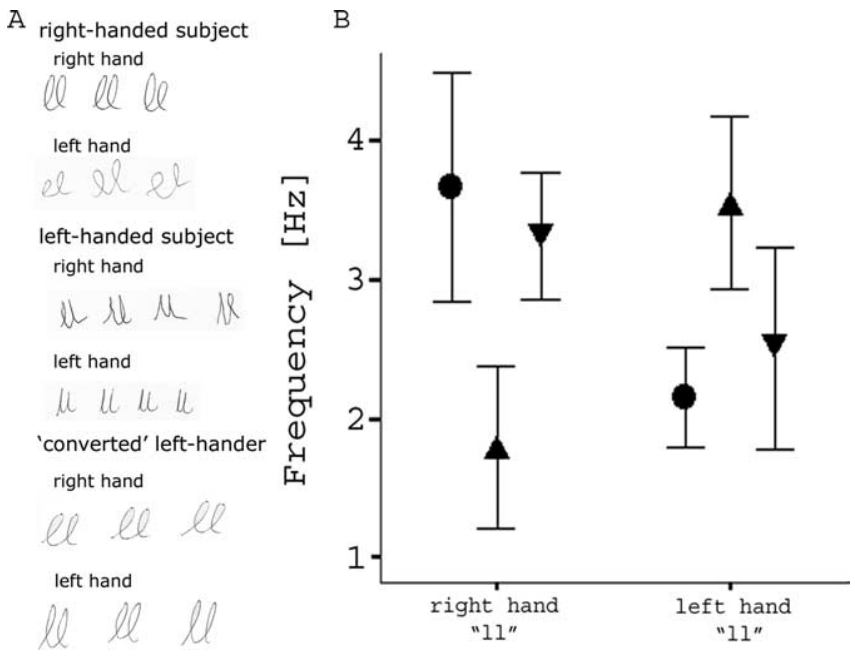

Figure 2. $\quad \boldsymbol{A}$, Examples from one subject per group writing lower case "II" on an electronic tablet. The converted left hander was able to write with both hands. $\boldsymbol{B}$, Writing kinematics. A comparison of frequencies writing "II" with both hands is shown. Error bars represent \pm 1 SD. "II" is writing two consecutive "I" letters. Circles, Right-handers; triangles, consistent left-handers; inverted triangles, converted left-handers.

right-hand stroke frequency were observed between consistent right-handers and converted left-handers. Accordingly, the laterality quotient for the right-to-left asymmetry in mean stroke frequency ranged from 1.45 to 2.16 (mean, 1.73 ; SD, \pm 0.22 ), from 0.31 to 0.73 (mean, $0.50 ; \mathrm{SD}, \pm 0.11$ ), and from 0.94 to 2.17 (mean, 1.40; SD, \pm 0.33 ) in right-handers, left-handers, and converted left-handers, respectively. Individual laterality quotients based on the Edinburgh handedness score and mean stroke frequency showed no correlation with each other in any of the three groups.

All participants found the choice reaction-time task easy with mean error rates below $1 \%$ in all groups. Using mean reaction time as a dependent variable, repeated-measures ANOVA showed a main effect of task $\left(F_{(1.78,30.13)}=13.97 ; p<0.001\right)$ attributable to longer mean reaction times with bilateral relative to right-sided button presses (Fig. $3 B$ ). There was also a task-byhandedness interaction $\left(F_{(3.56,80.13)}=3.18 ; p=0.02\right)$, indicating differential handedness-dependent task-related mean reaction times. However, post hoc reaction-time comparisons showed no mean reaction-time differences between converted left-handers and consistent right- or left-handers with any of the tasks (Fig. $3 B)$. We found no statistical difference in the laterality of first button press with the bilateral response task in any of the three groups.

\section{Imaging data}

Between-group comparisons identified two areas in the right hemisphere with effector-independent differences between all three groups (Fig. $4 A$ and supplemental Fig. S2, available at www. jneurosci.org as supplemental material). The first area was located in the supramarginal gyrus of the right inferior parietal lobule $(F=10.4 ; p=0.012 ; x, y, z$ coordinates: $63,-39,30)$; the second area was located in the ventrolateral part of the right dorsal premotor cortex (PMd) $(F=7.57 ; p=0.014 ; x, y, z$ coordinates: 48, 0, 57). Following-up on the ANOVA result, we explored these effector-independent differences in task-related neuronal activity in more detail. The plots of task-related activity in voxels displaying peaks of activation differences between the three groups showed that, in consistent left-handers, movement- related activity in right supramarginal gyrus and PMd lay between activation levels found in the other two groups (Fig. 4, bottom). Post hoc $t$ tests revealed significantly greater activations in both regions in converted left-handers compared with consistent left-handers and consistent right-handers. The $t$ and $p$ values for each post hoc comparison are listed in the supplemental material and supplemental Table S2, available at www.jneurosci.org.

Stepwise masking revealed no other component of the motor system that showed a significant difference in effectorindependent activity across tasks between any two of the three groups. There was no indication of a strengthening of effectorindependent motor representations in the (nondominant) left hemisphere in converted left-handers. Specifically, converted left-handers showed no effector-independent increases in taskrelated activation in the (nondominant) left hemisphere compared with individuals with consistent handedness (i.e., consistent right- or left-handers).

In contrast to categorical between-group comparisons, within-group regression analysis showed that, in converted lefthanders, task-related activity in the left primary sensorimotor hand area (SM1) and left caudal premotor cortex were associated with individual measures of dexterity (Fig. 5). Individual laterality quotients based on the Edinburgh handedness scale were correlated with task-related activity in the left SM1 $(t=3.25 ; p=$ $0.029 ; x, y, z$ coordinates: $-39,-30,69)$. The stronger the rightward shift in handedness, the greater the increase in task-related activity in this region. Task-related activity in the left caudal PMd showed a positive linear correlation with the laterality quotient based on mean writing stroke frequency $(t=3.18 ; p=0.042 ; x, y, z$ coordinates: $-36,-12,66)$. The stronger the right-hand advantage for mean stroke frequency, the greater the task-related activity in this region. The gradual increase in task-related activity in the left primary sensorimotor area and PMd with stronger "righthandedness" was present with both unimanual and bimanual button presses (Fig. 5).

\section{Discussion}

We found two effector-independent patterns of sensorimotor reorganization associated with a left-to-right switch in handwriting. In the left "nondominant" hemisphere, converted lefthanders showed an increase in movement-related activity in the SM1 and caudal PMd associated with the relative left-to-right shift in hand preference. In the right "dominant" hemisphere, converted left-handers showed greater movement-related activation of inferior parietal cortex and rostrolateral PMd compared with consistent right- and left-handers. The relative hyperactivity was uncorrelated with measures of the relative left-to-right shift in writing handedness.

Before we discuss the implications of these findings for current concepts of human handedness, we want to consider some methodological issues. We specifically included only converted left-handers who reported a clear innate preference for the left hand and underwent an educational left-to-right switch of writing. This inclusion criterion makes it very unlikely that we were looking simply at a group of innate right-handers or ambidextrous individuals who happened to start writing with the left hand. The degree of conversion varied across converted lefthanders. Hand movements less subject to educational influence tended to retain innate preferences for the left (dominant) hand (Fig. 1). This enabled us to test for regional differences in movement-related neuronal activity depending on the "magnitude" of the left-to-right shift. Interestingly, the laterality quotient obtained from the Edinburgh handedness score did not 
A

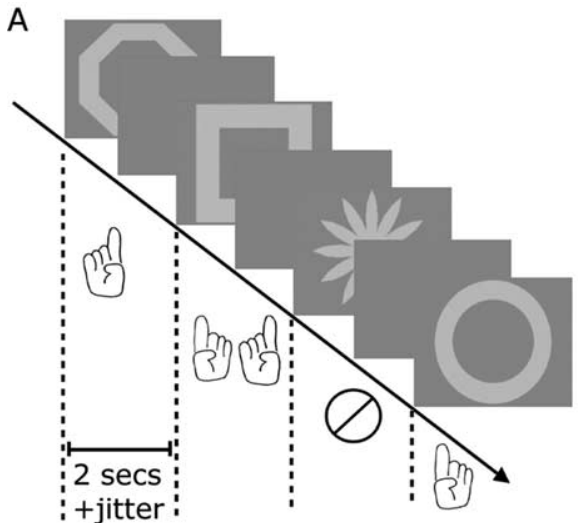

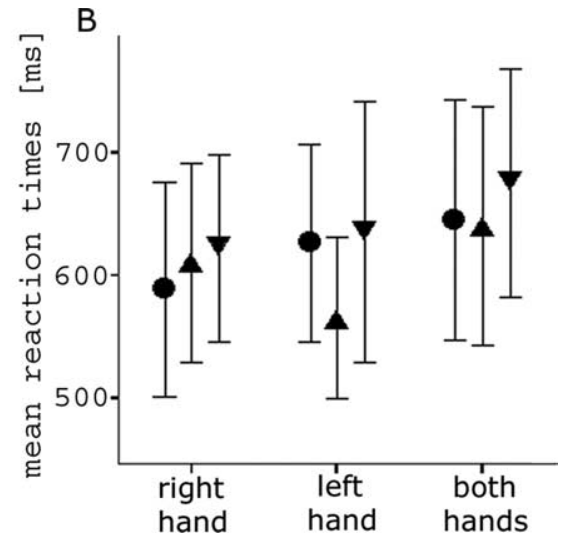

Figure 3. A, Schematic representation of the experimental paradigm. Each trial started with the presentation of a symbolic cue for $1 \mathrm{~s}$, followed by a dark gray screen. There were four different cues. Each cue prompted (1) a button press with the right index finger, (2) a button press with the left index finger, (3) a button press with both index fingers, or (4) no movement (no-go task). Subjects were instructed to respond as quickly as possible to the "go" cues and to refrain from any movement in response to the no-go cues. The time between presentations of two consecutive cues was jittered between 2.0 and $2.3 \mathrm{~s}$. The attribution of each symbol to a given task was varied across subjects. $\boldsymbol{B}$, Group differences for reaction time. First set consisted of a right-button press, the second set consisted of a left-button press, and the third set consisted of bilateral button presses. Circles, Right-handers; triangles, consistent left-handers; inverted triangles, converted left-handers.
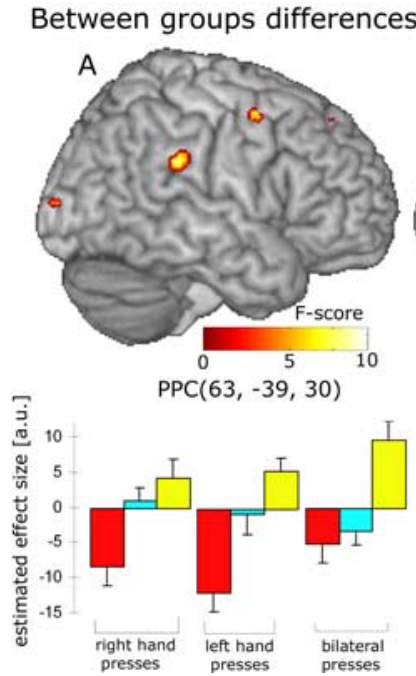
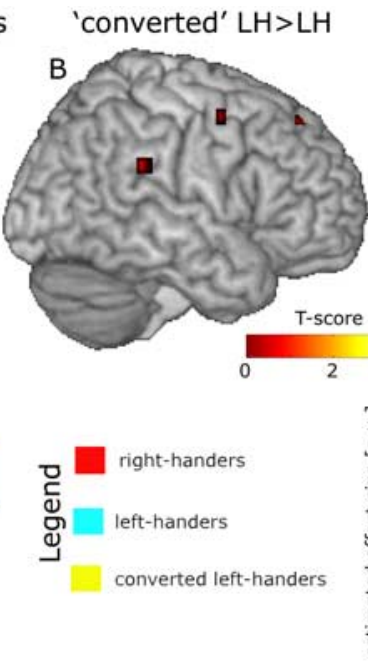

'converted' LH $>$ RH
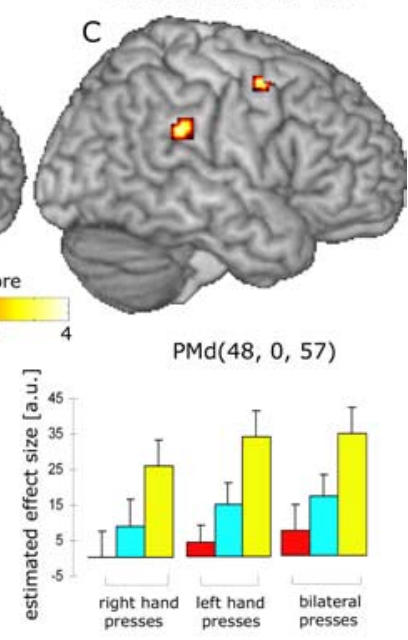

Figure 4. $\quad \boldsymbol{A}$, Areas showing a relative difference in movement-related BOLD signal increase over all movement conditions when comparing converted left-handers, consistent left-handers, and consistent right-handers with an $F$ test. $\boldsymbol{B}, \boldsymbol{C}$, Areas revealing greater activations in converted left-handers compared with consistent left-handers $(\boldsymbol{B})$ and consistent right-handers $(\boldsymbol{C})$ across all movement conditions. Activations rendered on a single subject in MNI space ( $p<0.01$, uncorrected; voxel extent threshold of 5). The surface-rendered activation maps were generated by using a sequential masking procedure (for details, see Materials and Methods). In the bottom right and left panels, the bars represent task-related BOLD signal changes as estimated within the general linear model. The parameter estimates are given for the voxel showing a peak difference in the betweengroups comparison. Error bars represent 1 SEM. A.U., Arbitrary units.

correlate with that based on stroke frequency during writing. This discrepancy suggests that both handedness measures probe complementary aspects of motor lateralization. This is not surprising because the laterality quotient of handwriting movements reflects differences in proficiency of a manual skill that had been switched. In contrast, the laterality quotients derived from the items of the Edinburgh handedness score (all with the same weight) reflects the efficacy of switching across a range of manual tasks.

Neuronal activity was probed during right, left, or bilateral button presses to test for effector-independent sensorimotor representations of handedness. By choosing simple movements, task performance was matched as closely as possible between hands and groups. Yet, performance was still influenced by handedness as indicated by an interaction between handedness and type of movement. This can be attributed to a tendency of consistent right-handers toward faster responses with unilateral right button presses. Conversely, consistent lefthanders tended to react faster with unilateral left responses. However, it is worth recalling that none of the movements showed consistent differences in mean reaction time between groups when each response type was directly compared. Therefore, we argue that movement -related activity in converted left-handers is not attributable to differences in task performance compared with consistent rightand left-handers.

The relative shift in handedness influenced task-related activity in left (nondominant) motor cortex. When we correlated task-related activity with degree of left-to-right shift in converted lefthanders, the left SM1 showed a linear correlation with right-hand advantage (as measured by the Edinburgh score). Taskrelated activity in the left caudal PMd increased with rightward advantage for writing, as indexed by the laterality index derived from handwriting kinematics. Critically, this positive correlation between neuronal activity and "success" of switching was not only found with righthand responses but also with left-hand and bimanual ones. Hence, effectorindependent increases in the contribution of left motor cortex to simple unimanual and bimanual finger movements represents a gradual shift of handedness from the (initially dominant) right to the (initially nondominant) left hemisphere.

Both the SM1 and caudal PMd are "executive" motor areas concerned with movement preparation and execution (Toni et al., 1999). Although activity in SM1 is closely linked to the basic kinematic parameters of movement (Blinkenberg et al., 1996; Sadato et al., 1997; Jäncke et al., 1998), caudal PMd is more implicated in motor preparation (Boussaoud, 2001; Picard and Strick, 2001). We propose that the neuronal correlates of handedness in these executive motor areas that we find and that have been previously reported in consistent right- and left-handers (Kim et al., 1993; Volkmann et al., 1998) are, at least in part, the consequence of which hand is preferred throughout life. As such, these representations are more susceptible to educationally induced modification designed to convert left-handed into right-handed writing. Left-handers need to systematically use their right hands across a variety of manual tasks to shift motor dominance to the initially nondominant hemisphere. According to this hypothesis, the correlation between task-related activity and measures of 
right-hand preference found in executive left-hemispheric motor cortex most likely reflects a long-term consequence of daily practice with the right (initially nondominant) hand. Our finding suggests that motor representations of simple finger movements are gradually strengthened in executive motor areas of the left (nondominant) hemisphere the more righthanded converted left-handers become, presumably as a result of use-dependent reorganization.

The correlation between task-related activity and degree of individual shift in handedness validates the approach of neuroimaging relatively simple motor tasks to probe neuronal correlates of handedness. A continuous relationship between degree of handedness and neuronal activation has previously been shown with unilateral motor tasks involving visually cued button presses (Dassonville et al., 1997; Singh et al., 1998). Our finding indicates that the attempt to switch handedness for writing has a wider impact on the functional neuroanatomy of the motor system that controls the hands, influencing even motor tasks that require little skill.

Although executive motor areas showed evidence in converted left-handers for a greater involvement of the left (nondominant) hemisphere in motor control over time, we also found evidence for the opposite. Between-group comparisons revealed that attempted handedness shifts to the (nondominant) right hand also leads to an effector-independent increase in movement-related activity in inferior parietal cortex and lateral PMd of the right (dominant) hemisphere. Our results are in agreement with activation patterns found during handwriting in converted left-handers (Siebner et al., 2002). When participants wrote with their right hands, converted left-handers exhibited greater activation of right-hemispheric frontoparietal areas than innate right-handers, with peak activations located in the left inferior parietal lobule and the lateral premotor cortex. Movement kinematics during handwriting were matched between groups. Converted left-handers also displayed a linear increase of task-related activity in right rostral supplementary motor area and right supramarginal gyrus with the degree of "residual" left-handedness as measured by the Edinburgh handedness score (Siebner et al., 2002). These findings show that cortical representations of writing movements persist in higher-order associative areas of the right (dominant) hemisphere despite switching during childhood. The persistence of these motor representations is stronger the less successfully manual skills have switched to the right hand. Our results confirm and extend these findings by showing that switching handwriting to the right nondominant hand also influences cortical motor representations of simple unilateral and bilateral finger movements with both right and left hands.

In consistent right-handers, the PMd and supramarginal gyrus of the (dominant) left hemisphere have been implicated in
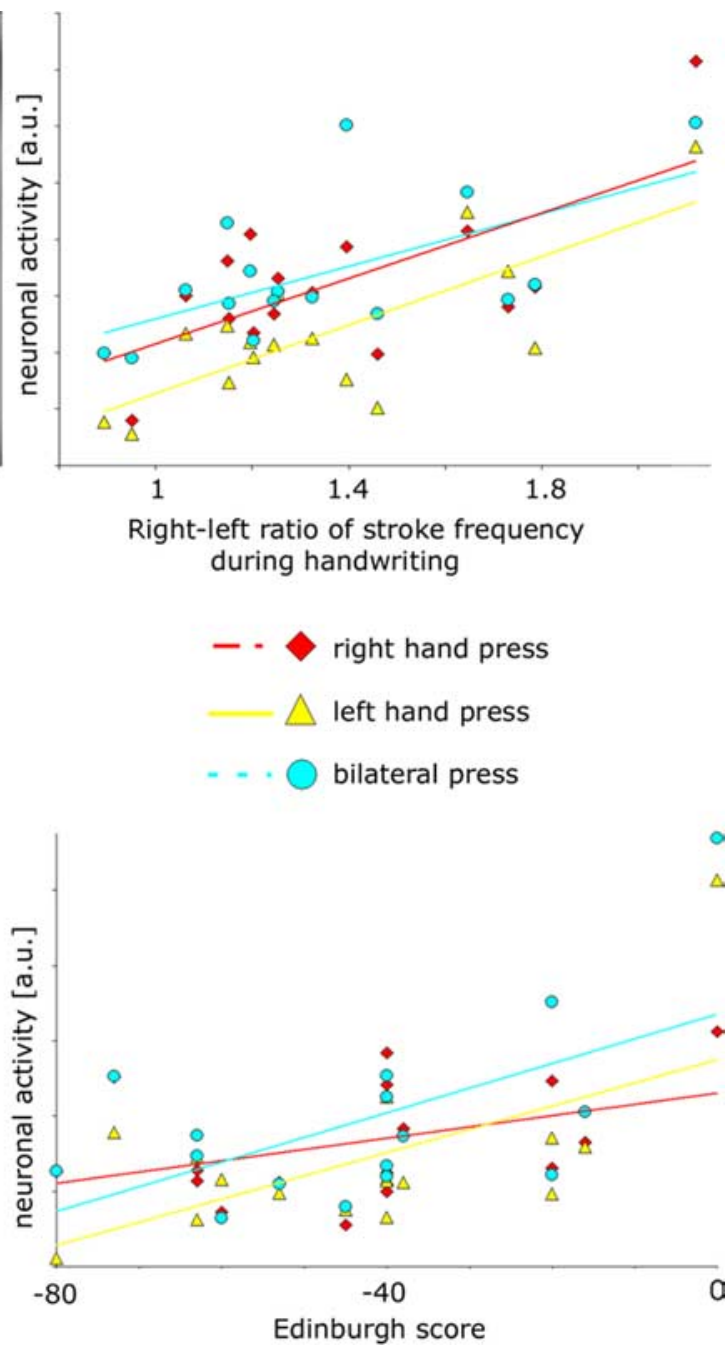

Edinburgh score

Figure 5. Correlation analysis for each of the three movements overlaid on a single subject's T1 image ( $p<0.01$, uncorrected). Areas showing greater activity with stronger right-handedness in the group of converted left-handers are shown. $A$ Correlation with quotient from writing performance. $\boldsymbol{B}$, Correlation with the Edinburgh Score. Red, Right-hand presses; yellow, left-hand presses; blue, bilateral presses; preG, precentral gyrus; posG, postcentral gyrus.

motor attention, movement preparation, and movement selection based on arbitrary cues (Deiber et al., 1996; Krams et al., 1998; Schlüter et al., 2001; Rushworth et al., 2003). Although few data are available on the functional significance of these regions for motor control in consistent left-handers, the homologous right-hemispheric areas are likely to play similar roles. Therefore, we argue that the increased activation of right PMd and supramarginal gyrus is related to higher-order aspects of motor control such as motor attention and preparation. This could be attributable to the fact that converted left-handers engage right (dominant)hemispheric activity to motor attention and preparation, even for the execution of very simple unilateral or bilateral finger movements.

Consistent unswitched left-handers have no brain regions with greater task-related activity than converted left-handers. Given the effector-independent increases of motor activation in the right lateral frontoparietal cortex in converted left-handers, we infer that attempts to switch handedness by educational training far from weakening the functional expression of lefthandedness in higher-order motor areas of the (dominant) right hemisphere in fact enhance it.

In summary, the reorganization patterns found in converted 
left-handers point to two distinct neuronal correlates of handedness in executive and associative sensorimotor cortices. Although the executive representations in SM1 and caudal PMd are more flexible and can be influenced by preferred hand use throughout life, representations in higher-order sensorimotor areas of the dominant hemisphere (i.e., in the inferior parietal cortex and rostrolateral $\mathrm{PMd}$ ) cannot be switched by educational training. Indeed, these higher-order representations are paradoxically strengthened by attempts to switch handedness for writing.

\section{References}

Amunts K, Schlaug G, Schleicher A, Steinmetz H, Dabringhaus A, Roland PE, Zilles K (1996) Asymmetry in the human motor cortex and handedness. NeuroImage 4:216-222.

Amunts K, Jäncke L, Mohlberg H, Steinmetz H, Zilles K (2000) Interhemispheric asymmetry of the human motor cortex related to handedness and gender. Neuropsychologia 38:304-312.

Annett M (1976) A coordination of hand preference and skill replicated. Br J Psychol 67:587-592.

Blinkenberg M, Bonde C, Holm S, Svarer C, Andersen J, Paulson OB, Law I (1996) Rate dependence of regional cerebral activation during performance of a repetitive motor task: a PET study. J Cereb Blood Flow Metab 16:794-803.

Boussaoud D (2001) Attention versus intention in the primate premotor cortex. NeuroImage 14:S40-S45.

Büchel C, Raedler T, Sommer M, Sach M, Weiller C, Koch MA (2004) White matter asymmetry in the human brain: a diffusion tensor MRI study. Cereb Cortex 14:945-951.

Corballis MC (2003) From mouth to hand: gesture, speech, and the evolution of right-handedness. Behav Brain Sci 26:199-208; discussion $208-160$.

Dassonville P, Zhu XH, Uurbil K, Kim SG, Ashe J (1997) Functional activation in motor cortex reflects the direction and the degree of handedness. Proc Natl Acad Sci USA 94:14015-14018.

Deiber MP, Ibanez V, Sadato N, Hallett M (1996) Cerebral structures participating in motor preparation in humans: a positron emission tomography study. J Neurophysiol 75:233-247.

Friston KJ, Frith CD, Turner R, Frackowiak RS (1995) Characterizing evoked hemodynamics with fMRI. NeuroImage 2:157-165.

Good CD, Johnsrude I, Ashburner J, Henson RN, Friston KJ, Frackowiak RS (2001) Cerebral asymmetry and the effects of sex and handedness on brain structure: a voxel-based morphometric analysis of 465 normal adult human brains. NeuroImage 14:685-700.

Herve PY, Mazoyer B, Crivello F, Perchey G, Tzourio-Mazoyer N (2005) Finger tapping, handedness and grey matter amount in the Rolando's genu area. NeuroImage 25:1133-1145.

Herve PY, Crivello F, Perchey G, Mazoyer B, Tzourio-Mazoyer N (2006) Handedness and cerebral anatomical asymmetries in young adult males. NeuroImage 29:1066-1079.

Jäncke L, Peters M, Schlaug G, Posse S, Steinmetz H, Muller-Gartner H (1998) Differential magnetic resonance signal change in human sensorimotor cortex to finger movements of different rate of the dominant and subdominant hand. Brain Res Cogn Brain Res 6:279-284.

Kim SG, Ashe J, Hendrich K, Ellermann JM, Merkle H, Ugurbil K, Georgo- poulos AP (1993) Functional magnetic resonance imaging of motor cortex: hemispheric asymmetry and handedness. Science 261:615-617.

Klöppel S, van Eimeren T, Glauche V, Vongerichten A, Munchau A, Frackowiak RS, Büchel C, Weiller C, Siebner HR (2007) The effect of handedness on cortical motor activation during simple bilateral movements. NeuroImage 34:274-280.

Krams M, Rushworth MF, Deiber MP, Frackowiak RS, Passingham RE (1998) The preparation, execution and suppression of copied movements in the human brain. Exp Brain Res 120:386-398.

Lutz K, Koeneke S, Wüstenberg T, Jäncke L (2005) Asymmetry of cortical activation during maximum and convenient tapping speed. Neurosci Lett 373:61-66.

Marquardt C, Mai N (1994) A computational procedure for movement analysis in handwriting. J Neurosci Methods 52:39-45.

Oldfield RC (1971) The assessment and analysis of handedness: the Edinburgh inventory. Neuropsychologia 9:97-113.

Picard N, Strick PL (2001) Imaging the premotor areas. Curr Opin Neurobiol 11:663-672.

Rushworth MF, Johansen-Berg H, Gobel SM, Devlin JT (2003) The left parietal and premotor cortices: motor attention and selection. NeuroImage 20 [Suppl 1]:S89-S100.

Sadato N, Yonekura Y, Waki A, Yamada H, Ishii Y (1997) Role of the supplementary motor area and the right premotor cortex in the coordination of bimanual finger movements. J Neurosci 17:9667-9674.

Schlüter ND, Krams M, Rushworth MF, Passingham RE (2001) Cerebral dominance for action in the human brain: the selection of actions. Neuropsychologia 39:105-113.

Serrien DJ, Ivry RB, Swinnen SP (2006) Dynamics of hemispheric specialization and integration in the context of motor control. Nat Rev Neurosci $7: 160-166$

Siebner HR, Limmer C, Peinemann A, Drzezga A, Bloem BR, Schwaiger M, Conrad B (2002) Long-term consequences of switching handedness: a positron emission tomography study on handwriting in "converted" lefthanders. J Neurosci 22:2816-2825.

Singh LN, Higano S, Takahashi S, Kurihara N, Furuta S, Tamura H, Shimanuki Y, Mugikura S, Fujii T, Yamadori A, Sakamoto M, Yamada S (1998) Comparison of ipsilateral activation between right and left handers: a functional MR imaging study. NeuroReport 9:1861-1866.

Solodkin A, Hlustik P, Noll DC, Small SL (2001) Lateralization of motor circuits and handedness during finger movements. Eur J Neurol 8:425-434.

Stucchi N, Viviani P (1993) Cerebral dominance and asynchrony between bimanual two-dimensional movements. J Exp Psychol Hum Percept Perform 19:1200-1220.

Sun T, Walsh CA (2006) Molecular approaches to brain asymmetry and handedness. Nat Rev Neurosci 7:655-662.

Toni I, Schlüter ND, Josephs O, Friston K, Passingham RE (1999) Signal-, set- and movement-related activity in the human brain: an event-related fMRI study. Cereb Cortex 9:35-49.

Volkmann J, Schnitzler A, Witte OW, Freund H (1998) Handedness and asymmetry of hand representation in human motor cortex. J Neurophysiol 79:2149-2154.

White LE, Lucas G, Richards A, Purves D (1994) Cerebral asymmetry and handedness. Nature 368:197-198.

Witelson SF, Kigar DL (1992) Sylvian fissure morphology and asymmetry in men and women: bilateral differences in relation to handedness in men. J Comp Neurol 323:326-340. 